\title{
New tricks of prolyl oligopeptidase inhibitors - A common drug therapy for several neurodegenerative diseases
}

\section{Svarcbahs, Reinis}

2019-03

Svarcbahs , R , Julku , U , Kilpelainen , T , Kyyrö , M , Jäntti , M \& Myohänen , T T 2019 , ' New tricks of prolyl oligopeptidase inhibitors - A common drug therapy for several neurodegenerative diseases ' , Biochemical Pharmacology , vol. 161 , pp. 113-120 . https://doi.org/10.1016/j.bcp.20

http://hdl.handle.net/10138/312566

https://doi.org/10.1016/j.bcp.2019.01.013

cc_by_nc_nd

acceptedVersion

Downloaded from Helda, University of Helsinki institutional repository.

This is an electronic reprint of the original article.

This reprint may differ from the original in pagination and typographic detail.

Please cite the original version. 
New tricks of prolyl oligopeptidase inhibitors - a common drug therapy for several neurodegenerative diseases

Reinis Svarcbahs, Ulrika Julku, Tommi Kilpeläinen, Mirva Kyyrö, Maria Jäntti, Timo T. Myöhänen

Division of Pharmacology and Pharmacotherapy / Drug Research Program

Faculty of Pharmacy

Viikinkaari 5E (PO Box 56)

00014 University of Helsinki

FINLAND

\section{Corresponding author:}

Dr. Timo Myöhänen

Division of Pharmacology and Pharmacotherapy / Drug Research Program

Faculty of Pharmacy, Viikinkaari 5E (PO Box 56), 00014 University of Helsinki, FINLAND

tel. $+358-50-5641426$

timo.myohanen@helsinki.fi 
Running title: Prolyl oligopeptidase as a drug target

Abstract: 239 words

Body text: 4810 words

Figures: 2

Tables: 1

References: 96

List of abbreviations: 1-methyl-4-phenyl-1,2,3,6-tetrahydropyridine, MPTP; adeno-associated virus, AAV; alpha-synuclein, aSyn; Alzheimer's disease, AD; amyloid-beta, A $\beta$; amyloid precursor peptide, APP; autophagy-lysosomal pathway, ALP; growth associated protein 43, GAP-43; Parkinson's disease, PD; Prolyl oligopeptidase, PREP; PREP mutant with S554A (inactive), S554A-PREP; Proteinase K, PK; reactive oxygen species, ROS; S129 phosphorylated aSyn, aSyn p-S129; thyrotropin-releasing hormone, TRH; ubiquitin-proteasomal system, UPS. 


\begin{abstract}
Changes in prolyl oligopeptidase (PREP) expression levels, protein distribution, and activity correlate with aging and are reported in many neurodegenerative conditions. Together with decreased neuropeptide levels observed in aging and neurodegeneration, and PREP's ability to cleave only small peptides, PREP was identified as a druggable target. Known PREP non-enzymatic functions were disregarded or attributed to PREP enzymatic activity, and several potent small molecule PREP inhibitors were developed during early stages of PREP research. These showed a lot of potential but variable results in experimental memory models, however, the initial excitement was short-lived and all of the clinical trials were discontinued in either Phase I or II clinical trials for unknown reasons. Recently, PREP's ability to form protein-protein interactions, alter cell proliferation and autophagy has gained more attention than earlier recognized catalytical activity. Of new findings, particularly the aggregation of alpha-synuclein (aSyn) that is seen in the presence of PREP is especially interesting because PREP inhibitors are capable of altering aSyn-PREP interaction in a manner that reduces the aSyn dimerization process. Therefore, it is possible that PREP inhibitors altering interactions should have different characteristics than those aimed for strong inhibition of catalytic activity. Moreover, PREP colocalization with aSyn and with tau and amyloid-beta hints to PREP's possible role not only in the synucleinopathies but in other neurodegenerative diseases as well. This commentary will focus on less well-acknowledged non-enzymatic functions of PREP that may provide a better approach for the development of PREP inhibitors for the treatment of neurodegenerative disorders.
\end{abstract}

Keywords: neurodegeneration, alpha-synuclein, Tau protein, amyloid-beta, autophagy, serine protease.

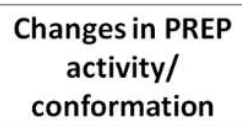

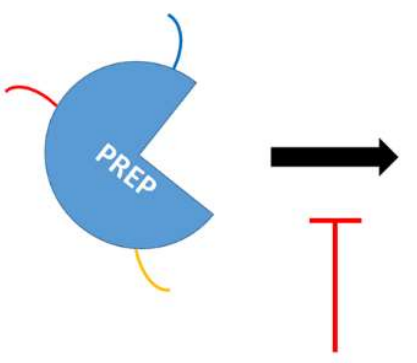

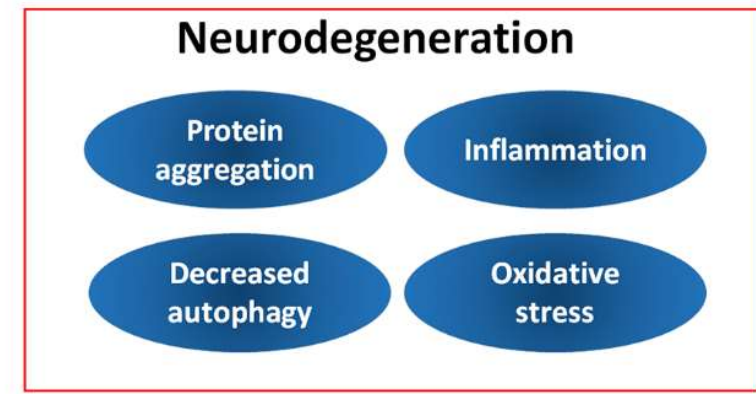

PREP inhibitor blocking the enzymatic activity/ induces conformational changes 


\section{Prolyl oligopeptidase (PREP) distribution and structure}

Prolyl oligopeptidase (PREP, also known as POP, PO or PEP; Fig 1) is a serine protease (EC 3.4.21.26) that is ubiquitously expressed throughout the body with the highest protein levels in brain, kidney, testis, and thymus, however, the expression levels do not necessarily correlate with the PREP enzymatic activity $[1,2]$ but this could be due the endogenous inhibition of PREP by $\alpha$-2-macroglobulin (Tenorio-Laranga et al. 2013 Biochem Pharm.) or due the precence of fibroblast activation protein (FAP) that cleaves similar substrates as PREP but is insensitive for PREP inhibitors (Collins et al. 2004 Int J Biochem Cell biol). Moreover, lack of good PREP antibodies has also made the verification of distribution studies difficult. In brain, the highest PREP protein expression and activity is seen in cortical and nigrostriatal tissues $[3,4]$ with almost exclusive localization in the neuronal cells [5]. Besides, high PREP activity is seen in proliferating cells in peripheral tissues and in different tumors [1]. mRNA level of PREP in rat brain is the highest during perinatal development, notably during cellular differentiation and neuronal migration, while later on during development PREP mRNA levels gradually decrease into adulthood [6]. However, during ageing PREP mRNA levels are again dramatically increased [7].

PREP is considered as evolutionary conserved enzyme that is present from archaeal species to human [9] although at least bacterial PREP has undergone several genetic modifications (Kaushik and Sowdhamini 2014). PREP was first identified as an oxytocin-cleaving enzyme from human uterine tissue [10] and later it was shown that catalytical PREP actions are exerted on the C-terminal side of the proline [11]. Initial crystallography studies with pig PREP demonstrated that PREP is a rigid structure composed of catalytical domain with $\alpha / \beta$ hydrolase fold and $\beta$-propeller domain (Fig. 1) $[12,13]$. Eukaryotic PREP has restrictive requirements for its substrate, peptides larger than approximately $3 \mathrm{kDa}$ (30 amino acids) cannot be cleaved by PREP [14] due to the position of a catalytical pocket between the catalytical and $\beta$ propeller domain [12]. Initially, the tunnel of the unusual $\beta$-propeller domain was thought to act as the 
access point for the substrate [13] but further studies have concluded that substrate access between PREP compartments is more likely $[15,16]$. In recent years, PREP shift in equilibrium between closed and open conformation has been demonstrated to occur, notably PREP inhibition shifting the protein conformation to a closed form where flexible loop structures that are located near the putative entry site to the catalytical site could be essential in gating peptide access (Fig. 1) [17]. [18]. (Li et al. JBC 2010). This was supported by the finding that mutations allowing more flexible movements for e.g. loop A increased catalytic activity of PREP but their truncations or stabilization decreased catalytic activity (Szeltner et al. 2013). Additionally, Kaszuba et al. (2012) suggested based on molecular dynamics study that also these loops are highly mobile during open and closed conformation of PREP, and PREP inhibitor binding will stabilize these movements, particularly loop A. Another molecular dynamics model (Kaushik et al. 2014) even suggested that catalytical and $\beta$-propeller domain twist and then open to allow substrates to enter the catalytical site. However, a recent study by Tsigirotagi et al. (2017) showed by using hydrogen/deuterium exchange mass spectrometry that PREP inhibitor binding stabilizes particularly B and His-loops but not that much loop A, and also stabilized the movement of catalytic site and $\beta$-propeller by allowing His-loop and $\beta$-propeller blade 1 to form bonds. Nevertheless, it is clear that PREP has active conformational dynamics and relevance of this for PREP functions has not been carefully characterized. 

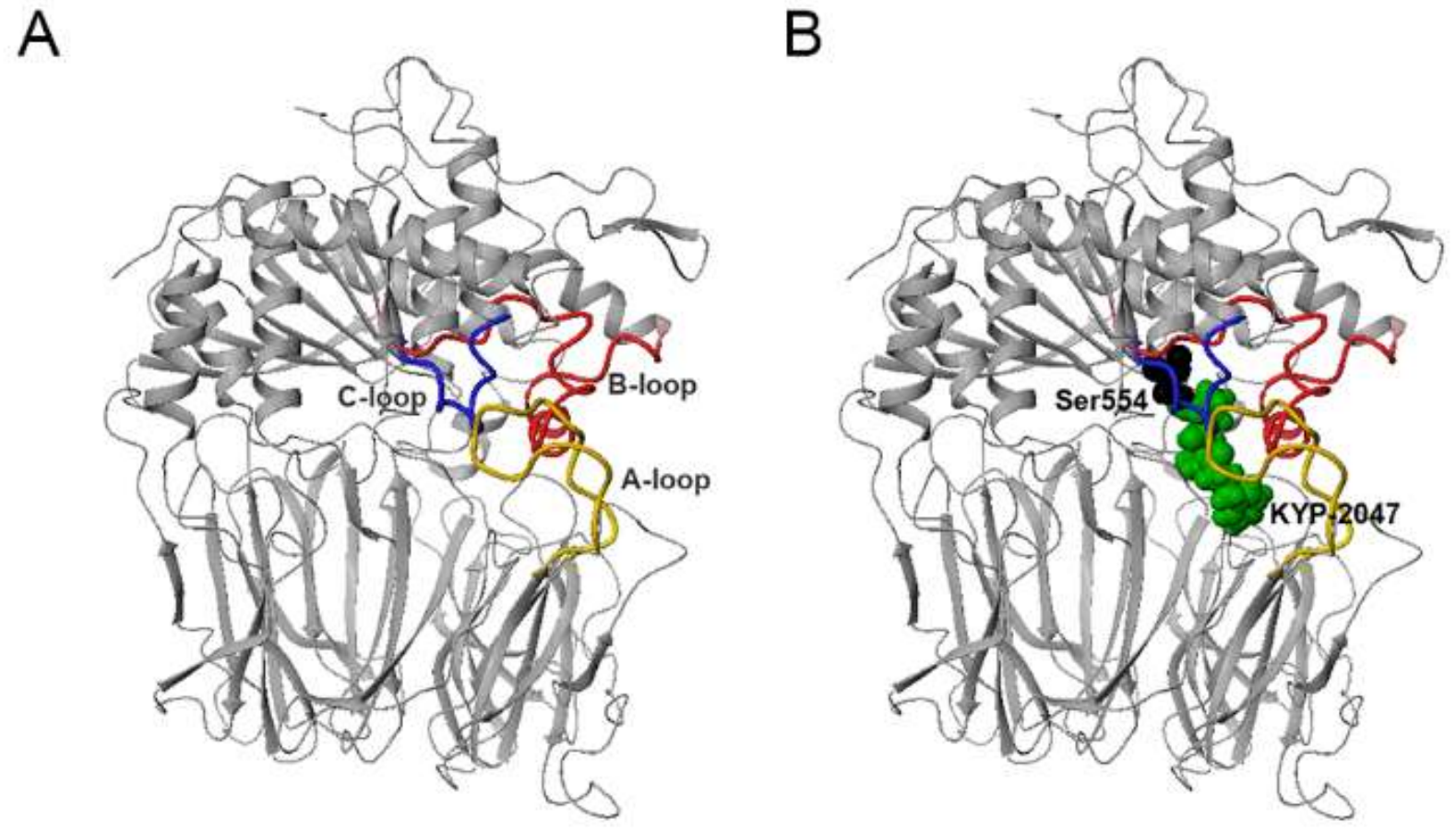

Figure 1A-B. The structure of PREP. PREP consists of two parts: catalytic and beta-propeller domain (A). Beta-propeller was thought to be the gating mechanism for substrates access to the active site, but later studies suggest that PREP is able to form openings between two domains, and that flexible loopstructures near the opening gate substrate access. Loops A (yellow), B (red) and C (blue) are colored in the figure A. Classical substrate-like inhibitors, such as KYP-2047 (marked green in the panel B), bind to the Ser554 (black) in the active site and stabilize the inactive "closed" conformation of PREP (B).

More recent studies have proposed that dynamics of PREP conformational changes could be taken into account to tailor PREP inhibitors that would more selectively affect either catalytical PREP activity or its protein-protein interaction $[17,19]$. Already in the study by Lopez et al. (2016 ChemBioChem) using multiple quantum relaxation dispersion assay, two rather similar PREP inhibitors, KYP-2047 and Z-Proprolinal had slightly different spectra on PREP. It is in line with recent reports of PREP inhibitors exhibiting different effects on cell differentiation. Researchers used two catalytical PREP inhibitors, KYP-2047 and SUAM-14746, but only the latter inhibited the differentiation of trophoblast stem cells, 
modelling placental development [20]. Consequently, it could be speculated that PREP catalytical inhibitors can exert varied impact on the PREP non-catalytical functions, namely due to subtle changes in the PREP conformation.

\section{Neurodegenerative diseases with prominent proteinopathies}

One of the most common features of neurodegenerative disorders are protein aggregation and accumulation. Parkinson's disease (PD) is characterized by the accumulation of aSyn aggregates that are a major component of Lewy bodies and Lewy neurites [21]. This hallmark is not exclusive to PD but is characteristic to all synucleinopathies, including dementia with Lewy bodies and multiple system atrophy [22]. On the other hand, the best-known Alzheimer's disease (AD) hallmarks are extracellular amyloidbeta $(A \beta)$ aggregates but recent failures on drug development against $A \beta$ have increased the interest on intracellular tau aggregation that is classically associated with dementia relater tauopathies, including AD. Both proteins share many characteristics as being partially unfolded proteins that under pathological conditions are prone to oligomerize and form insoluble aggregates [23].

Although largely debated which forms of aSyn are toxic, several studies suggest that intermediates formed during the aggregation process, aSyn oligomers and fibrils, are particularly toxic for dopaminergic neurons in substantia nigra, and can damage several cellular organelles, such as mitochondria to increase oxidative stress, and Golgi-ER and proteasomal processing to further increase aSyn aggregation. Additionally, aSyn fibrils can propagate by cell-to-cell transfer [24-26]. Similarly, elevated Tau phosphorylation leads to its release from microtubules, oligomerization and formation of neurofibrillary tangles $[27,28]$. As with aSyn, Tau oligomers can exit cells and propagate pathology, and extracellular aSyn and Tau has been shown to activate glial cells and cause increased neuroinflammation $[29,30]$. Moreover, protein aggregation in these disorders is not limited to the hallmark protein only; 
Tau aggregates are seen in PD and in dementia with Lewy bodies and aSyn accumulation can be seen in $\mathrm{AD}$ [31]. Additionally, these proteins can interact and enhance aggregation [32]. Taken together, targeting intracellular protein aggregates could be the pathway to develop disease-modifying therapies for several neurodegenerative diseases.

\section{Early studies on PREP in neurodegenerative diseases and PREP inhibitor development}

After Walter et al. (1971) discovered prolyl endopeptidase [10], for the next three decades only PREP enzymatic activity was known and considered during the PREP inhibitor development. Little attention was directed at varied effects of PREP inhibition that could not have been easily explained by catalytical inhibition of PREP, for extensive reviews see [33,34]. Consequently, strong emphasis was placed on potent PREP inhibitor development due to PREP's ability to cleave short proline-containing neuropeptides in vitro with a lot of research published on potential endogenous PREP substrates. Some of the most studied neuropeptides that apart from in vitro data have some indirect evidence of in vivo peptide modulation after PREP inhibition are substance P, arginine-vasopressin [35], and thyrotropinreleasing hormone (TRH) [36, 37]. Besides the aforementioned putative substrates, a comprehensive list of peptides linked to PREP catalysis from in vitro studies can be found in an earlier review by GarciaHorsman et al. 2007 [33].

Additionally, a possible link between PREP and amyloid precursor peptide (APP) processing was proposed. In the particular paper, short $\mathrm{C}$-terminal $\mathrm{A} \beta$ sequences were used to screen for the proteases capable of generating A $\beta$ from APP [38]. However, due to the size restrictions associated with PREP substrate [14] it is unlikely that PREP could cleave APP in vivo but Kato et al. (1997)[39] showed a significant decrease of $A \beta(1-16)$ fragments after PREP inhibitor, Y-29794, treatment in the senescence accelerated mouse brain. Although promising results, the mechanism remained unclear and there have 
been no further studies. It could be speculated that already cleaved short peptides originating from APP could be potential PREP substrates. However, peptidomics analyses have not yielded any conclusive results correlating putative PREP in vitro targets to ones obtained by peptide analyses from PREP inhibitor-treated animal tissue [40]. Additionally, decreased [41, 42] and increased [43] activity of PREP have been reported in the $\mathrm{AD}$ post mortem brains but this discrepancy could be attributed to the tissue collection and processing [33]. Another explanation could be the loss of neuronal cells in the assessed brain tissue samples as PREP is predominantly expressed in neurons and decreased activity could have arisen from loss of PREP rather than decrease in its activity [44]. PREP and A $\beta$ deposit colocalization has been demonstrated in the brains of senescence accelerated mouse [45] as well as in AD patients post mortem brain samples [46]. Moreover, it has been shown that PREP activity is decreased in the brains of PD, dementia with Lewy bodies, and Huntington's disease patients [41]. Decreased PREP activity has also been shown in PD patient cerebrospinal fluid samples [47]. However, these results should be interpreted with caution as post mortem tissue preservation and changes in redox state in tissue could have inactivated PREP $[33,48]$.

PREP has been implicated in the regulation of inositol cycle in social amoeba, Dictyostelium Discoideum [49], as well as in neuronal and glial cell lines where PREP inhibition was demonstrated to stimulate inositol production via substance $\mathrm{P}[50]$. The authors indicated that this would explain discrepancies between cellular PREP localization and apparent neuropeptide release in the extracellular space [50]. They hypothesized that PREP involvement in the inositol processing could explain in vivo PREP inhibitor effects associated with enhanced memory, learning, and neuroprotection [51].

Following either of the hypotheses, it was believed that PREP inhibition could ablate imbalance in peptidergic systems that arise due to ageing. Fittingly, initial animal studies showed promise: PREP inhibition by JTP-4819 was shown to improve memory in aged and middle cerebral artery-occluded rats. 
This effect was attributed to the increase in acetylcholine release and possibly decreased degradation of neuropeptides [52]. Additionally, PREP inhibition by S 17092 ameliorated cognitive decline in a monkey PD low-dose MPTP (1-methyl-4-phenyl-1,2,3,6-tetrahydropyridine) model. However, no histological evaluation of nigrostriatal tract or motor behavior in regard to MPTP impact on the dopaminergic neurons was described in this particular study [53]. Three PREP inhibitors, JTP-4819, Z-321, and S 17092, entered phase I and/or II clinical trials in late $90 \mathrm{~s}$ but shortly thereafter trials were discontinued. However, these trials demonstrated that small molecule PREP inhibitors can be safely administered in humans [5456]. A later report on $\mathrm{S} 17092$ explicitly stated that the pharmacodynamic parameters that were measured in phase I clinical trials did not allow determination of the therapeutic dose [57] pointing to dissatisfactory outcomes, most likely lack of cognitive improvement in Phase II clinical trials.

\section{Novel functions for PREP beyond hydrolytic actions}

\subsection{Protein-protein interactions}

After termination of PREP and cognition enhancing/AD related clinical trials, more focus has been directed at PREP non-enzymatic functions. It was shown in vitro in cell-free conditions that PREP coincubation with aSyn induced aggregation of this PD-related protein [58]. The effect was reversed by catalytical PREP inhibition but was not related to the ability of PREP to hydrolyze aSyn as this protein is much too large $(14 \mathrm{kDa})$ to be processed by PREP [58]. A year later, PREP interaction with growth associated protein 43 (GAP-43) was demonstrated (Table. 1) [59]. Of note was the observation that this association was independent of PREP enzymatic activity as catalytically inactive PREP mutant with S554A (S554A-PREP) substitution was able to form a protein-protein interaction with GAP-43. Moreover, it was shown that altered growth cone dynamics of PREP knockout (PREPko) neuronal cells 
could be rescued with either wild type or mutant S554A-PREP [59], further supporting the mechanismof-action for PREP beyond catalytic activity. The third protein-protein interaction partner of PREP currently published is glyceraldehyde 3-phosphate dehydrogenase (GAPDH, Table. 1) [60]. The authors postulated that this interaction is important for GAPDH nuclear translocation upon cell cycle arrest and apoptotic cell death in NB-1 neuroblastoma cells. Additionally, the effect could be blocked by PREP inhibition with SUAM-14746 or after PREP depletion [60]. In an earlier work, Z-pro-prolinal inhibition of PREP abolished GAPDH translocation in monkey fibroblast CV1-P cells but not in neuroblastoma SH-SY5Y cells [61]. Additionally, a yeast-two-hybrid and colocalization study suggested PREP interaction with tubulin and based on this, proposed role in cellular trafficking and axonal transport has as one of the functions for PREP [51]. However, this has not been verified in vitro or functional studies. Interestingly, we showed PREP colocalization with aSyn in PD brains as well as with A $\beta$ plaques and tau protein in $\mathrm{AD}$ patient post mortem brains, supporting the in vitro data of PREP interaction with aSyn. Additionally, increased immunostaining was seen in the astrocytes of AD patients [46]. Currently, known PREP protein interaction and non-enzymatic functions point to a more global PREP role in cellular signaling. A recent review has proposed that substrate binding and enzymatic activity could be secondary processes where PREP primarily functions through peptide gated direct interactions [62]. Whether the substrate binding is necessary or not, is still unclear. However, it can be speculated that the varied set of non-hydrolytic functions, such as protein-protein interactions, cellular pathway activation, and induction of cellular inflammation stem from unknown PREP mechanisms.

\subsection{PREP and neuroinflammation}

Although PREP is not visible in glial cells in the intact rodent brain, microglial activation by immunoglobulin G increased PREP expression [68]. A recent study has reported changes in the PREP 
protein activity after microglial stimulation with lipopolysaccharides (LPS) and IFN $\gamma$ in the absence or presence of neuronal cells. Notably, intracellular PREP activity decreased while PREP activity and protein in the extracellular space was increased. PREP inhibition reduced TNF $\alpha$ levels and exhibited neuroprotection in the KYP-2047 treatment groups [69]. Interestingly, it has been demonstrated that upon stimulation of TPH-1 monocytes by a combination of IFN $\gamma$ and either LPS or aSyn, enzymatic activity of PREP is upregulated. This increase in PREP activity could not be achieved by TPH-1 cell stimulation by only one of the inflammatory stimulators. Moreover, microglial supernatant toxicity stimulated by IFN $\gamma$ and aSyn in SH-SY5Y cells could be abolished by PREP inhibition [70]. This report is in line with a study where PREP knockout animals were shown to not respond to LPS-induced microglial activation [65] pointing to direct PREP involvement in neuroinflammation. Moreover, systemic inflammation in liver failure rat model (portacaval shunt) increased PREP expression, activity, and colocalization of PREP with astrocytes in several brain areas [71].

\subsection{PREP, PREP inhibition and aSyn aggregation in cells and in vivo}

\subsubsection{PREP interacts with aSyn to increase its aggregation}

Our group has done additional experiments on PREP and aSyn interactions, and we have confirmed PREP-aSyn interaction in vitro. Notably, we have shown that PREP increased aSyn dimerization by direct protein-protein interaction [64]. This effect is independent of PREP activity but only treatment of catalytically active PREP with a PREP inhibitor, KYP-2047, reduced aSyn dimerization while the S554A-PREP-mediated aSyn dimerization process remained unchanged. Earlier results (Myöhänen et al. 2012) suggested that PREP inhibition could reduce the interaction between PREP and aSyn but contrary to assumption, after KYP-2047 treatment, PREP-aSyn interaction increased pointing to the 
ability of KYP-2047-PREP to stabilize aSyn or bind aSyn in a way that restricts aSyn-aSyn interactions [64]. PREP in cells is thought to exist in different conformations [17], this can be seen on native gel electrophoresis where native PREP or S554A-PREP separates in to three distinctive bands believed to represent open and closed conformer and oligomer [64]. Whether oligomers exist in physiological conditions is not known. After PREP was incubated with an inhibitor, KYP-2047, only closed conformer was seen on the native gel, this effect was not reproduced with S554A-PREP [64]. Consequently, shift in the equilibrium between different PREP conformations could represent the beneficial effects that PREP exert on aSyn aggregation [64]. It will be important to establish if different PREP inhibitors affect PREP's non-catalytical functions differently and if this effect is due to changes in PREP conformation. It could be speculated that the PREP inhibitor's potency on enzymatic activity (IC50) is not the defining factor in the protein-protein interaction process.

\subsubsection{PREP inhibition enhances aSyn clearance by inducing autophagy}

It has been demonstrated that PREP inhibition reduces aSyn burden in cells and in vivo [72, 73]. This effect could not be solely explained by decreased aSyn aggregation, and we found that PREP inhibition not only reduces aSyn aggregation but also induces autophagy via class III phosphatidylinositol-4,5bisphosphate 3-kinase (PI3K-III) beclin1 pathway. This induction boosts autophagic machinery of the cell that in turn removes excessive aSyn from the cells [72] and also overcomes the effect of proteasomal inhibitor, lactacystin, on aSyn aggregation in a cell culture model [74]. However, the process is most likely more complex, as in the adeno-associated virus (AAV) aSyn overexpression mouse PD model where aSyn overexpression and subsequent chronic PREP inhibition had little effect on total aSyn immunoreactivity. It is likely that the aSyn load produced by AAV was high enough to not allow cellular protein clearance machinery to entirely degrade excess aSyn. However, PREP inhibition still had a 
beneficial effect on mouse motor behavior, which was associated with decrease in soluble higher aSyn oligomer counts in the PREP inhibitor treated animals [75]. Similarly, we had shown that soluble higher aSyn oligomers are reduced in aSyn overexpressing cells after PREP inhibition [73]. Proteinase K (PK)resistant aSyn inclusions were not decreased as efficiently as soluble forms after PREP inhibition in the mouse AAV aSyn overexpression model [75], and this suggests that it might be related to the inability of the protein degradation pathway to degrade PK-resistant aSyn inclusions after they have been formed. There are no reports about the ability of autophagy to degrade insoluble aSyn aggregates in vivo, and it is has been shown that preformed fibrils that generally are insoluble are not degraded by autophagy [76]. Whether Lewy bodies and higher order aSyn aggregates are the main cause of the neuronal toxicity is highly debated. Large aggregates could act as a protective mechanism that sequester soluble toxic oligomer species [77-79]. Moreover, there are reports showing that soluble oligomers rich in $\beta$-structure are highly toxic species [80], and this supports the use of PREP inhibition to reduce aSyn toxicity in synucleinopathies.

\subsubsection{PREP gives raise to soluble aSyn aggregates in cells and in vivo}

To further support the role of PREP for aSyn toxicity, we have recently shown that PREPko animals are less sensitive to aSyn overexpression caused by AAV-aSyn injection [81]. In the study, PREP overexpression in tandem with aSyn resulted in a more pronounced drop in locomotor activity in aSyn and PREP-injected animals. Cell culture data supported the finding of PREP potentiating aSyn toxicity, as aSyn and PREP co-transfected groups exhibited the highest cell death [81]. Additionally, PREPko mice that had PREP restored in their nigrostriatal tract did not exhibit any toxicity in another study [67]. Interestingly, PK-resistant aSyn oligomers were significantly reduced in PREPko mice injected with AAV-aSyn and PREP when compared to only the AAV-aSyn-injected group, and the same observation 
held true for S129 phosphorylated aSyn (aSyn p-S129) [81]. This could indicate that PREPko mice injected with aSyn and PREP AAVs have more soluble oligomer aSyn species that are thought to be more toxic [26, 82], and this is supported by our earlier findings of the PREP inhibitor in the AAV-aSyn mouse model (see above) [75]. Moreover, reduced aSyn p-S129 levels also corroborated this observation due to the fact that most of the aSyn in, for example, Lewy bodies are phosphorylated. However, whether p-S129 aSyn is an indicator of toxicity is not completely understood although it is widely used as a marker for aSyn toxicity [83]. We wanted to further characterize the impact of PREP on aSyn and did experiments with PREPko cells that similarly indicated that lack of PREP could ameliorate aSyn toxicity due to increased autophagic flux (Svarcbahs et al. 2018), thus supporting earlier PREP inhibitor findings on autophagy [72].

\subsection{PREP and the striatal dopaminergic system}

In addition to other neuroprotective mechanisms, PREP inhibitors are able to modulate nigrostriatal dopamine and the dopamine transporter. Jalkanen et al 2012 [84] showed that PREP inhibitors, KYP2047 and JTP-4819, reduce striatal extracellular dopamine in the rat brain and in our study KYP-2047 increased tissue concentration of dopamine in the striatum of A30P point-mutated transgenic mice [72]. However, KYP-2047 did not have significant effects on striatal extracellular dopamine or tissue concentration of dopamine in wild-type mice or AAV-aSyn injected mice, but inhibitor treatment elevated striatal extracellular concentration of dopamine metabolites in wild-type mice $[67,75]$. We have also shown that PREP participates in regulation of the dopamine transporter in mice $[67,72]$. Lack of PREP increases dopamine transporter phosphorylation and overexpression of PREP decreases it in the mouse striatum, but KYP-2047 treatment did not have an effect on dopamine transporter function [67]. Phosphorylated transporters are internalized which decreases them on the plasma membrane and reduces 
re-uptake of dopamine leading to an elevated extracellular dopamine concentration $[85,86]$. PREP has a significant role in regulating the nigrostriatal dopaminergic system but KYP-2047 has a limited beneficial effect on dopaminergic cells in mouse models of PD (Julku et al. 2018).

\section{Could PREP be a target for drug therapy in several neurodegenerative diseases?}

Our studies show that PREP interacts directly with aSyn and stabilizes a structure that is more prone to aggregate (Fig. 2) [64]. Interestingly, PREP does not appear in PD GWAS studies and its activity is more likely to be reduced than increased in PD post mortem brain samples and patient CSF (references), indicating that it is probably acting more as a catalyst than a cause for aSyn aggregation and this is supported by our results in cells and in vivo. Keeping in mind that only a small portion of all PD cases have a genetic background, PREP could be one of the factors behind sporadic PD. However, although not being crucial for aSyn aggregation, once aSyn concentration is increased or some other incident initiates the aSyn aggregation process, PREP can enhance the aggregation rate $[58,64]$. In cells and in vivo, the presence of PREP leads to formation of soluble aSyn oligomers that increases aSyn toxicity [81]. Although our early studies indicated that PREP inhibition could abolish this interaction [73], more detailed analysis showed that the PREP inhibitor changes and increases the interaction with aSyn, very likely stabilizing non-aggregating forms of aSyn, leading in part to significantly reduced oligomeric aSyn, improved cellular functions, and alleviated motor behavioral dysfunction in the mouse model. Interestingly, although PREP inhibitors were studied widely in AD and memory models, connection between PREP and Tau aggregation did not receive much attention. Only later studies revealed that PREP is localized in microtubules similar to Tau [51], and four-hour inhibition of PREP in rat CNS (S 17092 inhibitor) elevated levels of a small Tau peptide sequence [87]. Finally, our colocalization study showed similar spatial association between PREP and Tau in AD post mortem brain as with aSyn in PD post 
mortem samples [46]. Therefore, there is a possibility that PREP could be involved in the Tau aggregation process, but this particular hypothesis needs to be verified.

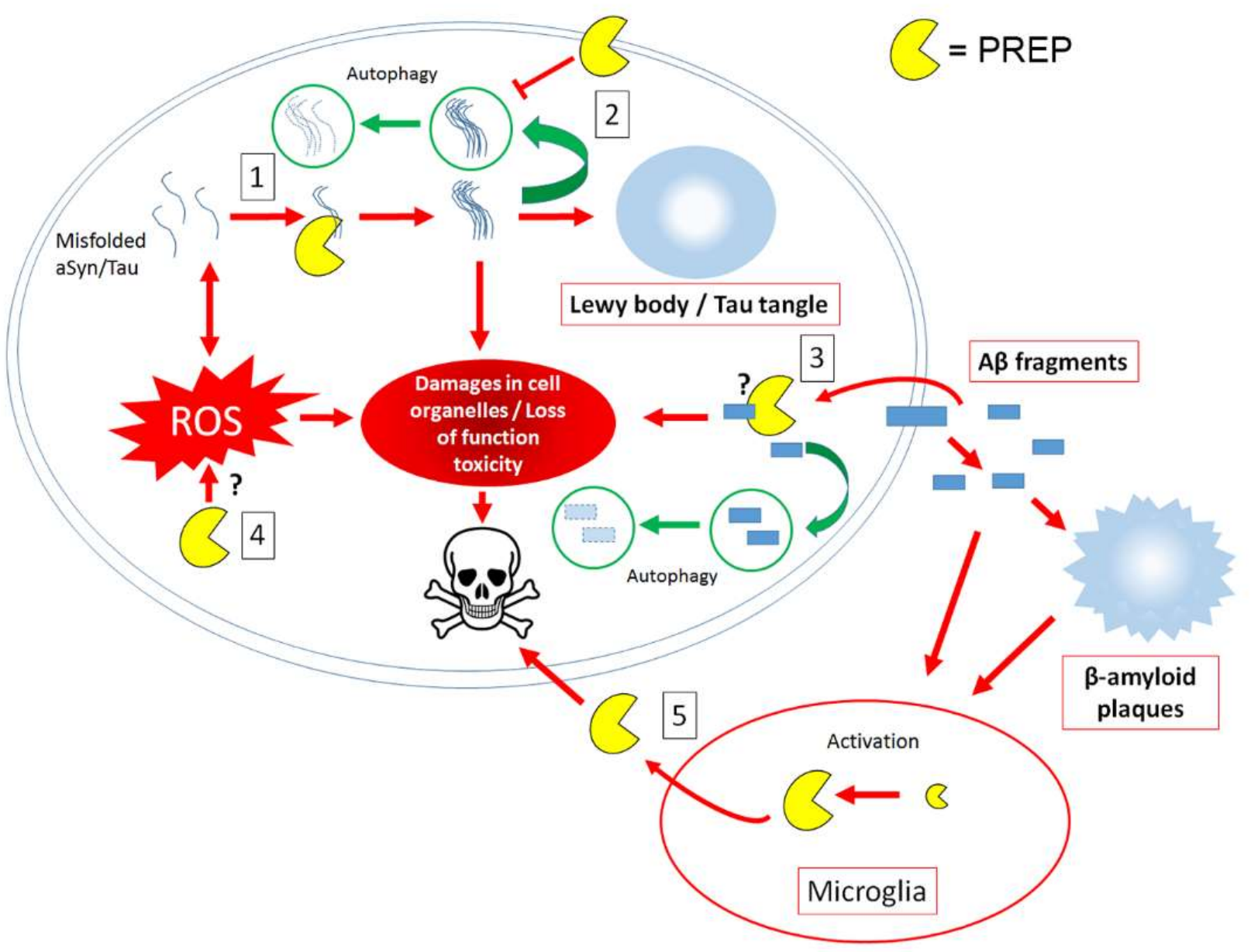

Figure 2. Known and putative PREP related pathways in neurodegeneration, and targets for PREP inhibitors. (1) PREP enhances aggregation and formation of soluble aSyn oligomers via direct interaction, and colocalization analysis indicates that this may also occur with Tau. PREP inhibitor modifies this interaction and stabilizes the less aggregation-prone conformation/conformer of aSyn. (2) PREP is a negative regulator for autophagy, and PREP inhibitor can enhance degradation of protein aggregates by enhancing autophagy. This can be beneficial in several neurodegenerative diseases. (3) PREP inhibitor treatment reduced $\mathrm{A} \beta$ fragments, and PREP colocalized with $\mathrm{A} \beta$ in $\mathrm{AD}$ post mortem brain. Therefore, it is a tempting possibility that PREP inhibitors could also modify intracellular A $\beta$ by regulating the interaction of $A \beta$ and PREP, and by enhancing their clearance via autophagy. (4) Increased ROS production is a common feature in several neurodegenerative diseases, and in a cellular model, PREP inhibition can reduce production of ROS. However, the role of PREP in oxidative stress and ROS production remains to be further studied. (5) A $\beta$ and aSyn oligomers can activate microglia, and activation increases PREP expression in microglia and leads to secretion of PREP in the extracellular space. This is potentially toxic for neurons, and PREP inhibitors can reduce extracellular PREP toxicity. 
Dysfunction in the protein degradation machinery, ubiquitin-proteasomal system (UPS), or autophagylysosomal pathway (ALP) has been implicated as one of the causes in most of the proteinopathies and has a distinctive role in the processing and aggregation of aSyn and Tau. Additionally, several gene mutations associated with $\mathrm{AD}$ (e.g. PICALM, PSEN1) and PD (e.g. SNCA, GBA) impair autophagy [88] and protein processing. Decreased levels of beclin1, an important regulator for autophagosome formation, have been measured in aging and $\mathrm{AD}$ [89]. Activation of these systems has been studied as a potential drug target that would alleviate protein aggregate accumulation [90]. It is not surprising that several studies have reported reduced protein accumulation and improved cognitive and motor behavior in models of AD, PD, Huntington's disease, and ALS after treatment by an autophagy inducer, such as rapamycin, or by increasing autophagy proteins like beclin1 (see comprehensive review by Galluzzi et al. 2017)[91]. Interestingly, autophagy induction e.g. by lithium and nilotinib, also lowers the levels of aggregation-prone $\mathrm{A} \beta 1-42$ in the brain of $\mathrm{AD}$ animal models $[92,93]$, indicating that their impact is not only limited to intracellular aggregates. It is possible that this was the mechanism behind the decrease of A $\beta$-fragment levels in senescence mouse model after PREP inhibitor treatment as reported by Kato et al. (1997) [39]. However, most autophagy inducers, including rapamycin, were not initially developed as autophagy inducers and have either an unclear mechanism-of-action or side effects that limit their clinical use in neurodegeneration. Additionally, autophagy regulates processes leading to apoptosis and safe regulation of these processes has been a challenge [91]. Our results show that PREP inhibition increases autophagy and elevates the levels of beclin1 [63] that has been reported to be depleted e.g. in AD, and there are no reports of PREP inhibition toxicity in preclinical and clinical trials [54-56]. Therefore, this could increase the usability of PREP inhibitors outside PD and they could be used generally in neurodegenerative diseases. 
Oxidative stress is one of the common toxic features in neurodegeneration. Mitochondrial damage and production of reactive oxygen species (ROS) increase the aggregation of aggregation-prone proteins, but oligomers and other aggregation intermediates can also damage mitochondria to increase ROS production and oxidative stress. We did observe reduced ROS production by a PREP inhibitor in rotenone-treated SH-SY5Y cells overexpressing aSyn but this was more related to decreased aSyn oligomers [94]. However, our recent paper [81] revealed that lack of PREP completely abolishes ROS production and an increase in stress response proteins after oxidative stress, therefore it could be hypothesized that PREP is a factor in cellular redox regulation. Puttonen et al. showed in 2006 that a PREP inhibitor, Z-Pro-Prolinal, reduced oxidative stress in CV1-P cells and this impact was connected to GAPDH translocation [61]. However, GAPDH is a downstream target for ROS, and thus this cannot explain why PREP deletion leads to a significantly reduced stress response in cells. If PREP inhibitors could combat oxidative stress by reducing ROS production, it would further support the multi-target character of PREP inhibition. However, PREPko cells showed reduced 20S proteasomal activity at basal conditions but this can be a compensatory mechanism for increased autophagic flux since in transgenic aSyn mice PREP inhibition was shown to restore $20 \mathrm{~S}$ proteasomal activity to the wild-type mice levels $[72,81]$.

\section{Future directions}

As shown above, PREP is involved in several common mechanisms of toxicity related to neuronal death in neurodegenerative diseases. However, apart from aSyn, impact of PREP and its inhibition on other aggregation-prone proteins related to neurodegeneration has not been studied in detail, and requires further studies. Additionally, discrepancy between studies using different PREP inhibitors in models of neurodegeneration or memory loss (reviewed in Männistö et al. 2007 [62]), has never been carefully studied and this could reveal critical information on the mechanism of action of PREP inhibitors. In this 
review, we have mainly focused on the role of PREP in neurodegeneration. A simple cleavage of peptides cannot explain most of the actions associated to PREP. However, PREP inhibition has a potential, for example, to reduce cancer proliferation rate. Namely, neuroblastoma, gastric cancer, and human breast cancer cell lines have been shown to be sensitive to PREP inhibition. The effects arise from the $\mathrm{G}_{0} / \mathrm{G}_{1}$ arrest but the mechanism has remained unclear $[95,96]$. Moreover, different potent PREP inhibitors exhibit varied potency on the non-hydrolytic PREP functions, such as modulation of ROS production, cell differentiation, and inhibition of cancer cell proliferation, thus pointing on fine changes in PREP structure induced by these inhibitors. If this holds true, the possibility to develop inhibitors that could be specifically tailored for non-enzymatic PREP activities could be an exciting prospect for PREP inhibitor development for neurodegenerative disorders. However, this warrants firstly the identification of PREP structure (or structures) responsible for these interactions and identification of PREP inhibitor scaffolds and their binding sites in PREP that modify this structure and PREP conformation. There is still work ahead with PREP but its potential in drug discovery should not be forgotten.

\section{Acknowledgements}

The authors want to thank Dr. Mari Savolainen for her comments on the manuscript and PREP research. Studies behind the commentary have been supported by Academy of Finland (grants 303833, 267788, 273799, 318327, 305710), Jane and Aatos Erkko Foundation, Sigrid Juselius Foundation, Finnish Parkinson's Disease Foundation and University of Helsinki Grants for Timo T. Myöhänen. Authors state no conflicts of interests. 


\section{References}

[1] F. Goossens, I. De Meester, G. Vanhoof, S. Scharpe, Distribution of prolyl oligopeptidase in human peripheral tissues and body fluids, Eur. J. Clin. Chem. Clin. Biochem. 34(1) (1996) 17-22.

[2] T.T. Myohanen, J.I. Venalainen, J.A. Garcia-Horsman, M. Piltonen, P.T. Mannisto, Distribution of prolyl oligopeptidase in the mouse whole-body sections and peripheral tissues, Histochem Cell Biol 130(5) (2008) 993-1003.

[3] J. Irazusta, G. Larrinaga, J. González-Maeso, J. Gil, J.J. Meana, L. Casis, Distribution of prolyl endopeptidase activities in rat and human brain, Neurochem. Int. 40(4) (2002) 337-345.

[4] T.T. Myöhänen, J.I. Venäläinen, E. Tupala, J.A. Garcia-Horsman, R. Miettinen, P.T. Männistö, Distribution of Immunoreactive Prolyl Oligopeptidase in Human and Rat Brain, Neurochem. Res. 32(8) (2007) 1365-1374.

[5] S. Rossner, I. Schulz, U. Zeitschel, R. Schliebs, V. Bigl, H.U. Demuth, Brain prolyl endopeptidase expression in aging, APP transgenic mice and Alzheimer's disease, Neurochem Res 30(6-7) (2005) 695-702.

[6] N. Agirregoitia, P. Bizet, E. Agirregoitia, I. Boutelet, L. Peralta, H. Vaudry, S. Jegou, Prolyl endopeptidase mRNA expression in the central nervous system during rat development, J Chem Neuroanat 40(1) (2010) 53-62. [7] C.H. Jiang, J.Z. Tsien, P.G. Schultz, Y. Hu, The effects of aging on gene expression in the hypothalamus and cortex of mice, Proc Natl Acad Sci U S A 98(4) (2001) 1930-4.

[8] G. Bellemère, H. Vaudry, L. Mounien, I. Boutelet, S. Jégou, Localization of the mRNA encoding prolyl endopeptidase in the rat brain and pituitary, The Journal of Comparative Neurology 471(2) (2004) 128-143.

[9] J.I. Venalainen, R.O. Juvonen, P.T. Mannisto, Evolutionary relationships of the prolyl oligopeptidase family enzymes, Eur J Biochem 271(13) (2004) 2705-15.

[10] R. Walter, H. Shlank, J.D. Glass, I.L. Schwartz, T.D. Kerenyi, Leucylglycinamide released from oxytocin by human uterine enzyme, Science 173(3999) (1971) 827-9.

[11] M. Koida, R. Walter, Post-proline cleaving enzyme. Purification of this endopeptidase by affinity chromatography, J. Biol. Chem. 251(23) (1976) 7593-7599.

[12] V. Fulop, Z. Bocskei, L. Polgar, Prolyl oligopeptidase: an unusual beta-propeller domain regulates proteolysis, Cell 94(2) (1998) 161-70.

[13] V. Fulop, Z. Szeltner, L. Polgar, Catalysis of serine oligopeptidases is controlled by a gating filter mechanism, EMBO Rep 1(3) (2000) 277-81.

[14] A. Moriyama, M. Nakanishi, M. Sasaki, Porcine muscle prolyl endopeptidase and its endogenous substrates, J. Biochem. 104(1) (1988) 112-7.

[15] Z. Szeltner, D. Rea, T. Juhász, V. Renner, V. Fülöp, L. Polgár, Concerted Structural Changes in the Peptidase and the Propeller Domains of Prolyl Oligopeptidase are Required for Substrate Binding, J. Mol. Biol. 340(3) (2004) 627-637.

[16] T. Juhász, Z. Szeltner, V. Fülöp, L. Polgár, Unclosed $\beta$-Propellers Display Stable Structures: Implications for Substrate Access to the Active Site of Prolyl Oligopeptidase, J. Mol. Biol. 346(3) (2005) 907-917.

[17] A. Lopez, F. Herranz-Trillo, M. Kotev, M. Gairi, V. Guallar, P. Bernado, O. Millet, T. Tarrago, E. Giralt, ActiveSite-Directed Inhibitors of Prolyl Oligopeptidase Abolish Its Conformational Dynamics, ChemBioChem 17(10) (2016) 913-7.

[18] Z. Szeltner, T. Juhász, I. Szamosi, D. Rea, V. Fülöp, K. Módos, L. Juliano, L. Polgár, The loops facing the active site of prolyl oligopeptidase are crucial components in substrate gating and specificity, Biochim. Biophys. Acta 1834(1) (2013) 98-111.

[19] N. Kichik, T. Tarrago, B. Claasen, M. Gairi, O. Millet, E. Giralt, 15N relaxation NMR studies of prolyl oligopeptidase, an $80 \mathrm{kDa}$ enzyme, reveal a pre-existing equilibrium between different conformational states, ChemBioChem 12(18) (2011) 2737-9.

[20] Y. Maruyama, S. Matsubara, A.P. Kimura, Mouse prolyl oligopeptidase plays a role in trophoblast stem cell differentiation into trophoblast giant cell and spongiotrophoblast, Placenta 53 (2017) 8-15. 
[21] M.G. Spillantini, M.L. Schmidt, V.M. Lee, J.Q. Trojanowski, R. Jakes, M. Goedert, Alpha-synuclein in Lewy bodies, Nature 388(6645) (1997) 839-40.

[22] M.G. Spillantini, M. Goedert, The $\alpha$-Synucleinopathies: Parkinson's Disease, Dementia with Lewy Bodies, and Multiple System Atrophy, Ann. N. Y. Acad. Sci. 920(1) (2000) 16-27.

[23] S. Moussaud, D.R. Jones, E.L. Moussaud-Lamodière, M. Delenclos, O.A. Ross, P.J.J.M.N. McLean, Alphasynuclein and tau: teammates in neurodegeneration?, 9(1) (2014) 43.

[24] E. Angot, J.A. Steiner, C.M. Lema Tomé, P. Ekström, B. Mattsson, A. Björklund, P. Brundin, Alpha-Synuclein Cell-to-Cell Transfer and Seeding in Grafted Dopaminergic Neurons In Vivo, PLoS One 7(6) (2012) e39465.

[25] K.C. Luk, V. Kehm, J. Carroll, B. Zhang, P. O’Brien, J.Q. Trojanowski, V.M.Y. Lee, Pathological $\alpha$-Synuclein Transmission Initiates Parkinson-like Neurodegeneration in Non-transgenic Mice, Science (New York, N.Y.) 338(6109) (2012) 949-953.

[26] B. Winner, R. Jappelli, S.K. Maji, P.A. Desplats, L. Boyer, S. Aigner, C. Hetzer, T. Loher, M. Vilar, S. Campioni, C. Tzitzilonis, A. Soragni, S. Jessberger, H. Mira, A. Consiglio, E. Pham, E. Masliah, F.H. Gage, R. Riek, In vivo demonstration that al pha-synuclein oligomers are toxic, Proc Natl Acad Sci U S A 108(10) (2011) 4194-9.

[27] M. Goedert, S. Satumtira, R. Jakes, M.J. Smith, C. Kamibayashi, C.L. White, 3rd, E. Sontag, Reduced binding of protein phosphatase $2 \mathrm{~A}$ to tau protein with frontotemporal dementia and parkinsonism linked to chromosome 17 mutations, J Neurochem 75(5) (2000) 2155-62.

[28] M.G. Spillantini, M. Goedert, Tau pathology and neurodegeneration, Lancet Neurol. 12(6) (2013) 609-22.

[29] C.E.G. Leyns, D.M. Holtzman, Glial contributions to neurodegeneration in tauopathies, Mol. Neurodegener. 12(1) (2017) 50-50.

[30] D. Brück, G.K. Wenning, N. Stefanova, L. Fellner, Glia and alpha-synuclein in neurodegeneration: A complex interaction, Neurobiol. Dis. 85 (2016) 262-274.

[31] W.R. Galpern, A.E. Lang, Interface between tauopathies and synucleinopathies: a tale of two proteins, Ann. Neurol. 59(3) (2006) 449-58.

[32] X. Li, S. James, P. Lei, Interactions Between alpha-Synuclein and Tau Protein: Implications to Neurodegenerative Disorders, J Mol Neurosci 60(3) (2016) 298-304.

[33] J.A. Garcia-Horsman, P.T. Mannisto, J.I. Venalainen, On the role of prolyl oligopeptidase in health and disease, Neuropeptides 41(1) (2007) 1-24.

[34] J. Lawandi, S. Gerber-Lemaire, L. Juillerat-Jeanneret, N. Moitessier, Inhibitors of Prolyl Oligopeptidases for the Therapy of Human Diseases: Defining Diseases and Inhibitors, J. Med. Chem. 53(9) (2010) 3423-3438.

[35] K. Toide, K. Okamiya, Y. Iwamoto, T. Kato, Effect of a novel prolyl endopeptidase inhibitor, JTP-4819, on prolyl endopeptidase activity and substance P- and arginine-vasopressin-like immunoreactivity in the brains of aged rats, J Neurochem 65(1) (1995) 234-40.

[36] M. Shinoda, K. Okamiya, K. Toide, Effect of a novel prolyl endopeptidase inhibitor, JTP-4819, on thyrotropin-releasing hormone-like immunoreactivity in the cerebral cortex and hippocampus of aged rats, Jpn. J. Pharmacol. 69(3) (1995) 273-6.

[37] G. Bellemere, H. Vaudry, P. Morain, S. Jegou, Effect of prolyl endopeptidase inhibition on argininevasopressin and thyrotrophin-releasing hormone catabolism in the rat brain, J Neuroendocrinol $17(5)(2005)$ 306-13.

[38] S. Ishiura, T. Tsukahara, T. Tabira, T. Shimizu, K. Arahata, H. Sugita, Identification of a putative amyloid A4generating enzyme as a prolyl endopeptidase, FEBS Lett. 260(1) (1990) 131-134.

[39] A. Kato, A. Fukunari, Y. Sakai, T. Nakajima, Prevention of amyloid-like deposition by a selective prolyl endopeptidase inhibitor, Y-29794, in senescence-accelerated mouse, J. Pharmacol. Exp. Ther. 283(1) (1997) 328-35.

[40] J. Tenorio-Laranga, P.T. Mannisto, M. Storvik, P. Van der Veken, J.A. Garcia-Horsman, Four day inhibition of prolyl oligopeptidase causes significant changes in the peptidome of rat brain, liver and kidney, Biochimie 94(9) (2012) 1849-59. 
[41] D. Mantle, G. Falkous, S. Ishiura, P.J. Blanchard, E.K. Perry, Comparison of proline endopeptidase activity in brain tissue from normal cases and cases with Alzheimer's disease, Lewy body dementia, Parkinson's disease and Huntington's disease, Clin. Chim. Acta 249(1-2) (1996) 129-139.

[42] C. Ichai, N. Chevallier, P. Delaere, P. Dournaud, J. Epelbaum, J.J. Hauw, J.P. Vincent, F. Checler, Influence of region-specific alterations of neuropeptidase content on the catabolic fates of neuropeptides in Alzheimer's disease, J Neurochem 62(2) (1994) 645-55.

[43] T. Aoyagi, T. Wada, M. Nagai, F. Kojima, S. Harada, T. Takeuchi, H. Takahashi, K. Hirokawa, T. Tsumita, Deficiency of kallikrein-like enzyme activities in cerebral tissue of patients with alzheimer's disease, Experientia 46(1) (1990) 94-97.

[44] K.S. Laitinen, T. van Groen, H. Tanila, J. Venalainen, P.T. Mannisto, I. Alafuzoff, Brain prolyl oligopeptidase activity is associated with neuronal damage rather than beta-amyloid accumulation, Neuroreport 12(15) (2001) 3309-12.

[45] A. Fukunari, A. Kato, Y. Sakai, T. Yoshimoto, S. Ishiura, K. Suzuki, T. Nakajima, Colocalization of prolyl endopeptidase and amyloid beta-peptide in brains of senescence-accelerated mouse, Neurosci Lett 176(2) (1994) 201-4.

[46] M.J. Hannula, T.T. Myohanen, J. Tenorio-Laranga, P.T. Mannisto, J.A. Garcia-Horsman, Prolyl oligopeptidase colocalizes with alpha-synuclein, beta-amyloid, tau protein and astroglia in the post-mortem brain samples with Parkinson's and Alzheimer's diseases, Neuroscience 242 (2013) 140-50.

[47] M. Hagihara, T. Nagatsu, Post-proline cleaving enzyme in human cerebrospinal fluid from control patients and parkinsonian patients, Biochem. Med. Metab. Biol. 38(3) (1987) 387-91.

[48] T. Tsukahara, S. Ishiura, H. Sugita, Regulation of prolyl endopeptidase activity by the intracellular redox state, 265(35) (1990) 21448-53.

[49] R.S. Williams, M. Eames, W.J. Ryves, J. Viggars, A.J. Harwood, Loss of a prolyl oligopeptidase confers resistance to lithium by elevation of inositol $(1,4,5)$ trisphosphate, EMBO J 18(10) (1999) 2734-45.

[50] I. Schulz, B. Gerhartz, A. Neubauer, A. Holloschi, U. Heiser, M. Hafner, H.-U. Demuth, Modulation of inositol 1,4,5-triphosphate concentration by prolyl endopeptidase inhibition, Eur. J. Biochem. 269(23) (2002) 5813-5820.

[51] I. Schulz, U. Zeitschel, T. Rudolph, D. Ruiz-Carrillo, J.-U. Rahfeld, B. Gerhartz, V. Bigl, H.-U. Demuth, S. Roßner, Subcellular localization suggests novel functions for prolyl endopeptidase in protein secretion, J. Neurochem. 94(4) (2005) 970-979.

[52] K. Toide, M. Shinoda, Y. Iwamoto, T. Fujiwara, K. Okamiya, A. Uemura, A novel prolyl endopeptidase inhibitor, JTP-4819, with potential for treating Alzheimer's disease, Behav Brain Res 83(1-2) (1997) 147-51.

[53] J.S. Schneider, M. Giardiniere, P. Morain, Effects of the prolyl endopeptidase inhibitor S 17092 on cognitive deficits in chronic low dose MPTP-treated monkeys, Neuropsychopharmacology 26(2) (2002) 176-82.

[54] P. Morain, J.L. Robin, G. De Nanteuil, R. Jochemsen, V. Heidet, D. Guez, Pharmacodynamic and pharmacokinetic profile of $S 17092$, a new orally active prolyl endopeptidase inhibitor, in elderly healthy volunteers. A phase I study, Br. J. Clin. Pharmacol. 50(4) (2000) 350-9.

[55] K. Umemura, K. Kondo, Y. Ikeda, T. Kobayashi, Y. Urata, M. Nakashima, Pharmacokinetics and safety of JTP4819 , a novel specific orally active prolyl endopeptidase inhibitor, in healthy male volunteers, Br. J. Clin. Pharmacol. 43(6) (1997) 613-8.

[56] K. Umemura, K. Kondo, Y. Ikeda, M. Nishimoto, Y. Hiraga, Y. Yoshida, M. Nakashima, Pharmacokinetics and safety of Z-321, a novel specific orally active prolyl endopeptidase inhibitor, in healthy male volunteers, J. Clin. Pharmacol. 39(5) (1999) 462-70.

[57] P. Morain, P. Lestage, G. De Nanteuil, R. Jochemsen, J.L. Robin, D. Guez, P.A. Boyer, S 17092: a prolyl endopeptidase inhibitor as a potential therapeutic drug for memory impairment. Preclinical and clinical studies, CNS Drug Rev 8(1) (2002) 31-52. 
[58] I. Brandt, M. Gerard, K. Sergeant, B. Devreese, V. Baekelandt, K. Augustyns, S. Scharpe, Y. Engelborghs, A.M. Lambeir, Prolyl oligopeptidase stimulates the aggregation of alpha-synuclein, Peptides 29(9) (2008) 14728.

[59] E. Di Daniel, C.P. Glover, E. Grot, M.K. Chan, T.H. Sanderson, J.H. White, C.L. Ellis, K.T. Gallagher, J. Uney, J. Thomas, P.R. Maycox, A.W. Mudge, Prolyl oligopeptidase binds to GAP-43 and functions without its peptidase activity, Mol Cell Neurosci 41(3) (2009) 373-82.

[60] T. Matsuda, M. Sakaguchi, S. Tanaka, T. Yoshimoto, M. Takaoka, Prolyl oligopeptidase is a glyceraldehyde3-phosphate dehydrogenase-binding protein that regulates genotoxic stress-induced cell death, Int J Biochem Cell Biol 45(4) (2013) 850-7.

[61] K.A. Puttonen, S. Lehtonen, A. Raasmaja, P.T. Mannisto, A prolyl oligopeptidase inhibitor, Z-Pro-Prolinal, inhibits glyceraldehyde-3-phosphate dehydrogenase translocation and production of reactive oxygen species in CV1-P cells exposed to 6-hydroxydopamine, Toxicol In Vitro 20(8) (2006) 1446-54.

[62] P.T. Mannisto, J.A. Garcia-Horsman, Mechanism of Action of Prolyl Oligopeptidase (PREP) in Degenerative Brain Diseases: Has Peptidase Activity Only a Modulatory Role on the Interactions of PREP with Proteins?, Front. Aging Neurosci. 9(27) (2017) 27.

[63] M. Savolainen, kirjoittaja, The effects of prolyl oligopeptidase inhibition in $\alpha$-synuclein based mouse models of Parkinson's disease, Dissertationes scholae doctoralis ad sanitatem investigandam Universitatis Helsinkiensis, ISSN 2342-3161, University of Helsinki, Helsinki, 2015.

[64] M.H. Savolainen, X. Yan, T.T. Myohanen, H.J. Huttunen, Prolyl oligopeptidase enhances alpha-synuclein dimerization via direct protein-protein interaction, J Biol Chem 290(8) (2015) 5117-26.

[65] C. Hofling, N. Kulesskaya, K. Jaako, I. Peltonen, P.T. Mannisto, A. Nurmi, N. Vartiainen, M. Morawski, A. Zharkovsky, V. Voikar, S. Rossner, J.A. Garcia-Horsman, Deficiency of prolyl oligopeptidase in mice disturbs synaptic plasticity and reduces anxiety-like behaviour, body weight, and brain volume, Eur. Neuropsychopharmacol. 26(6) (2016) 1048-61.

[66] M.J. Moreno-Baylach, K.A. Puttonen, J. Tenorio-Laranga, J.I. Venalainen, M. Storvik, M.M. Forsberg, J.A. Garcia-Horsman, Prolyl endopeptidase is involved in cellular signalling in human neuroblastoma SH-SY5Y cells, Neurosignals 19(2) (2011) 97-109.

[67] U.H. Julku, A.E. Panhelainen, S.E. Tiilikainen, R. Svarcbahs, A.E. Tammimaki, T.P. Piepponen, M.H. Savolainen, T.T. Myohanen, Prolyl Oligopeptidase Regulates Dopamine Transporter Phosphorylation in the Nigrostriatal Pathway of Mouse, Mol Neurobiol 55(1) (2018) 470-482.

[68] A. Penttinen, J. Tenorio-Laranga, A. Siikanen, M. Morawski, S. Rossner, J.A. Garcia-Horsman, Prolyl oligopeptidase: a rising star on the stage of neuroinflammation research, CNS Neurol. Disord. Drug Targets 10(3) (2011) 340-8.

[69] T.A. Natunen, M. Gynther, H. Rostalski, K. Jaako, A.J. Jalkanen, Extracellular prolyl oligopeptidase derived from activated microglia is a potential neuroprotection target, Basic Clin. Pharmacol. Toxicol. (2018).

[70] A. Klegeris, J. Li, T.K. Bammler, J. Jin, D. Zhu, D.T. Kashima, S. Pan, S. Hashioka, J. Maguire, P.L. McGeer, J. Zhang, Prolyl endopeptidase is revealed following SILAC analysis to be a novel mediator of human microglial and THP-1 cell neurotoxicity, Glia 56(6) (2008) 675-685.

[71] J. Tenorio-Laranga, C. Montoliu, A. Urios, V. Hernandez-Rabaza, H. Ahabrach, J.A. García-Horsman, V. Felipo, The expression levels of prolyl oligopeptidase responds not only to neuroinflammation but also to systemic inflammation upon liver failure in rat models and cirrhotic patients, J. Neuroinflammation 12 (2015) 183.

[72] M.H. Savolainen, C.T. Richie, B.K. Harvey, P.T. Mannisto, K.A. Maguire-Zeiss, T.T. Myohanen, The beneficial effect of a prolyl oligopeptidase inhibitor, KYP-2047, on alpha-synuclein clearance and autophagy in A30P transgenic mouse, Neurobiol. Dis. 68 (2014) 1-15.

[73] T.T. Myohanen, M.J. Hannula, R. Van Elzen, M. Gerard, P. Van Der Veken, J.A. Garcia-Horsman, V. Baekelandt, P.T. Mannisto, A.M. Lambeir, A prolyl oligopeptidase inhibitor, KYP-2047, reduces alpha-synuclein 
protein levels and aggregates in cellular and animal models of Parkinson's disease, Br J Pharmacol 166(3) (2012) 1097-113.

[74] T.T. Myöhänen, S. Norrbacka, M.H. Savolainen, Prolyl oligopeptidase inhibition attenuates the toxicity of a proteasomal inhibitor, lactacystin, in the alpha-synuclein overexpressing cell culture, Neurosci. Lett. 636 (2017) 83-89.

[75] R. Svarcbahs, U.H. Julku, T.T. Myohanen, Inhibition of Prolyl Oligopeptidase Restores Spontaneous Motor Behavior in the alpha-Synuclein Virus Vector-Based Parkinson's Disease Mouse Model by Decreasing alphaSynuclein Oligomeric Species in Mouse Brain, J Neurosci 36(49) (2016) 12485-12497.

[76] S.A. Tanik, C.E. Schultheiss, L.A. Volpicelli-Daley, K.R. Brunden, V.M.Y. Lee, Lewy Body-like $\alpha$-Synuclein Aggregates Resist Degradation and Impair Macroautophagy, The Journal of Biological Chemistry 288(21) (2013) 15194-15210.

[77] P. Brundin, K.D. Dave, J.H. Kordower, Therapeutic approaches to target alpha-synuclein pathology, Exp. Neurol. 298 (2017) 225-235.

[78] C. Peng, R.J. Gathagan, V.M. Lee, Distinct alpha-Synuclein strains and implications for heterogeneity among alpha-Synucleinopathies, Neurobiol. Dis. 109(Pt B) (2018) 209-218.

[79] H.L. Roberts, D.R. Brown, Seeking a mechanism for the toxicity of oligomeric alpha-synuclein, Biomolecules 5(2) (2015) 282-305.

[80] N. Cremades, Samuel I.A. Cohen, E. Deas, Andrey Y. Abramov, Allen Y. Chen, A. Orte, M. Sandal, Richard W. Clarke, P. Dunne, Francesco A. Aprile, Carlos W. Bertoncini, Nicholas W. Wood, Tuomas P.J. Knowles,

Christopher M. Dobson, D. Klenerman, Direct Observation of the Interconversion of Normal and Toxic Forms of a-Synuclein, Cell 149(5) (2012) 1048-1059.

[81] R. Svarcbahs, U.H. Julku, S. Norrbacka, T.T. Myohanen, Removal of prolyl oligopeptidase reduces alphasynuclein toxicity in cells and in vivo, Sci. Rep. 8(1) (2018) 1552.

[82] D.P. Karpinar, M.B. Balija, S. Kugler, F. Opazo, N. Rezaei-Ghaleh, N. Wender, H.Y. Kim, G. Taschenberger, B.H. Falkenburger, H. Heise, A. Kumar, D. Riedel, L. Fichtner, A. Voigt, G.H. Braus, K. Giller, S. Becker, A. Herzig, M. Baldus, H. Jackle, S. Eimer, J.B. Schulz, C. Griesinger, M. Zweckstetter, Pre-fibrillar alpha-synuclein variants with impaired beta-structure increase neurotoxicity in Parkinson's disease models, EMBO J 28(20) (2009) 325668.

[83] A. Oueslati, Implication of Alpha-Synuclein Phosphorylation at S129 in Synucleinopathies: What Have We Learned in the Last Decade?, J. Parkinsons Dis. 6(1) (2016) 39-51.

[84] A.J. Jalkanen, T.P. Piepponen, J.J. Hakkarainen, I. De Meester, A.-M. Lambeir, M.M. Forsberg, The effect of prolyl oligopeptidase inhibition on extracellular acetylcholine and dopamine levels in the rat striatum, Neurochem. Int. 60(3) (2012) 301-309.

[85] R.A. Vaughan, R.A. Huff, G.R. Uhl, M.J.J.J.o.B.C. Kuhar, Protein kinase C-mediated phosphorylation and functional regulation of dopamine transporters in striatal synaptosomes, 272(24) (1997) 15541-15546.

[86] H.E. Melikian, K.M.J.J.o.n. Buckley, Membrane trafficking regulates the activity of the human dopamine transporter, 19(18) (1999) 7699-7710.

[87] W.M. Nolte, D.M. Tagore, W.S. Lane, A. Saghatelian, Peptidomics of prolyl endopeptidase in the central nervous system, Biochemistry 48(50) (2009) 11971-11981.

[88] F.M. Menzies, A. Fleming, A. Caricasole, C.F. Bento, S.P. Andrews, A. Ashkenazi, J. Fullgrabe, A. Jackson, M. Jimenez Sanchez, C. Karabiyik, F. Licitra, A. Lopez Ramirez, M. Pavel, C. Puri, M. Renna, T. Ricketts, L. Schlotawa, M. Vicinanza, H. Won, Y. Zhu, J. Skidmore, D.C. Rubinsztein, Autophagy and Neurodegeneration: Pathogenic Mechanisms and Therapeutic Opportunities, Neuron 93(5) (2017) 1015-1034.

[89] F. Pickford, E. Masliah, M. Britschgi, K. Lucin, R. Narasimhan, P.A. Jaeger, S. Small, B. Spencer, E.

Rockenstein, B. Levine, T. Wyss-Coray, The autophagy-related protein beclin 1 shows reduced expression in early Alzheimer disease and regulates amyloid beta accumulation in mice, J. Clin. Invest. 118(6) (2008) 2190-9. [90] D. Ebrahimi-Fakhari, L. Wahlster, P.J. McLean, Protein degradation pathways in Parkinson's disease: curse or blessing, Acta Neuropathol 124(2) (2012) 153-72. 
[91] L. Galluzzi, E.H. Baehrecke, A. Ballabio, P. Boya, J.M. Bravo-San Pedro, F. Cecconi, A.M. Choi, C.T. Chu, P. Codogno, M.I. Colombo, A.M. Cuervo, J. Debnath, V. Deretic, I. Dikic, E.L. Eskelinen, G.M. Fimia, S. Fulda, D.A. Gewirtz, D.R. Green, M. Hansen, J.W. Harper, M. Jaattela, T. Johansen, G. Juhasz, A.C. Kimmelman, C. Kraft, N.T. Ktistakis, S. Kumar, B. Levine, C. Lopez-Otin, F. Madeo, S. Martens, J. Martinez, A. Melendez, N. Mizushima, C. Munz, L.O. Murphy, J.M. Penninger, M. Piacentini, F. Reggiori, D.C. Rubinsztein, K.M. Ryan, L. Santambrogio, L. Scorrano, A.K. Simon, H.U. Simon, A. Simonsen, N. Tavernarakis, S.A. Tooze, T. Yoshimori, J. Yuan, Z. Yue, Q. Zhong, G. Kroemer, Molecular definitions of autophagy and related processes, EMBO J 36(13) (2017) 18111836.

[92] A. Fiorentini, M.C. Rosi, C. Grossi, I. Luccarini, F. Casamenti, Lithium improves hippocampal neurogenesis, neuropathology and cognitive functions in APP mutant mice, PLoS One 5(12) (2010) e14382.

[93] M.L. Hebron, I. Lonskaya, C.E. Moussa, Nilotinib reverses loss of dopamine neurons and improves motor behavior via autophagic degradation of alpha-synuclein in Parkinson's disease models, Hum. Mol. Genet. 22(16) (2013) 3315-28.

[94] L. Dokleja, M.J. Hannula, T.T. Myohanen, Inhibition of prolyl oligopeptidase increases the survival of alphasynuclein overexpressing cells after rotenone exposure by reducing alpha-synuclein oligomers, Neurosci Lett 583 (2014) 37-42.

[95] M. Sakaguchi, T. Matsuda, E. Matsumura, T. Yoshimoto, M. Takaoka, Prolyl oligopeptidase participates in cell cycle progression in a human neuroblastoma cell line, Biochem Biophys Res Commun 409(4) (2011) 693-8. [96] S. Tanaka, K. Suzuki, M. Sakaguchi, The prolyl oligopeptidase inhibitor SUAM-14746 attenuates the proliferation of human breast cancer cell lines in vitro, Breast Cancer 24(5) (2017) 658-666. 